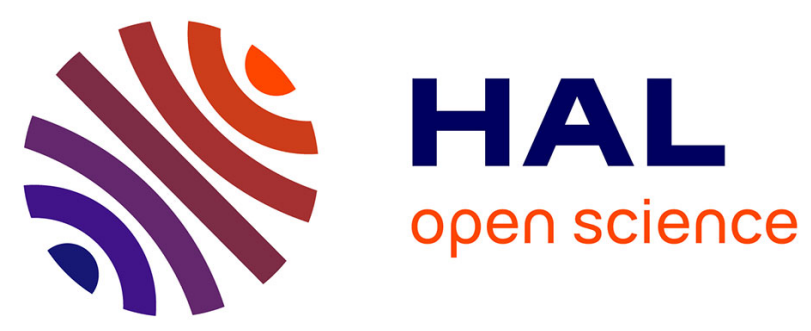

\title{
Modélisation électrique des canalisations métalliques des bâtiments : application aux réseaux de capteurs intelligents
}

\author{
Denis Pellerin, M. Brissaud, G. Grange, D. Noterman, J.F. Robin
}

\section{- To cite this version:}

Denis Pellerin, M. Brissaud, G. Grange, D. Noterman, J.F. Robin. Modélisation électrique des canalisations métalliques des bâtiments : application aux réseaux de capteurs intelligents. Revue de Physique Appliquée, 1989, 24 (9), pp.933-944. 10.1051/rphysap:01989002409093300 . jpa-00246129

HAL Id: jpa-00246129

https://hal.science/jpa-00246129

Submitted on 1 Jan 1989

HAL is a multi-disciplinary open access archive for the deposit and dissemination of scientific research documents, whether they are published or not. The documents may come from teaching and research institutions in France or abroad, or from public or private research centers.
L'archive ouverte pluridisciplinaire HAL, est destinée au dépôt et à la diffusion de documents scientifiques de niveau recherche, publiés ou non, émanant des établissements d'enseignement et de recherche français ou étrangers, des laboratoires publics ou privés. 


\title{
Modélisation électrique des canalisations métalliques des bâtiments : application aux réseaux de capteurs intelligents
}

\author{
D. Pellerin ( $\left.{ }^{1}\right)$, M. Brissaud $\left({ }^{1}\right)$, G. Grange $\left({ }^{1}\right)$, D. Noterman $\left({ }^{1}\right)$ et J. F. Robin $\left({ }^{2}\right)$ \\ (1) Laboratoire de Génie Electrique et Ferroélectricité, I.N.S.A. Lyon, 20 avenue Albert Einstein, \\ 69621 Villeurbanne Cedex. France \\ ${ }^{2}$ ) Centre de Recherche ELF Solaize, BP 22, 69360 Saint Symphorien d'Ozon, France
}

(Reçu le 28 novembre 1988, révisé le 21 avril 1989, accepté le 30 mai 1989)

\begin{abstract}
Résumé. - La commande de processus, la gestion de l'énergie et la surveillance des bâtiments existants nécessitent l'utilisation de capteurs qui pour fonctionner et échanger des informations doivent être alimentés et interconnectés. La pose de conducteurs d'alimentation et la réalisation physique des interconnexions sont des opérations coûteuses. Nous proposons d'utiliser les réseaux de canalisations métalliques des bâtiments comme unique support de transmission et d'alimentation. Un modèle électrique à base de quadripôles est proposé pour décrire le processus d'alimentation à distance, ou téléalimentation, des capteurs. Il permet de prévoir les performances du support de transmission. Les résultats expérimentaux obtenus sur site réel confirment la validité du modèle.

Abstract. - Process control, energy management and monitoring of existing buildings need sensors which have to be supplied and connected for running and interchanging data. The installation of wires or cables and the actual achievement of the connections are generally expensive. The use of the metallic pipe networks of buildings for both the information transmission and supply is proposed. An electric model based on quadripole approach is proposed for describing the remote supply process of sensors. It allows to forecast the performances of this new transmission support. Experimental and theoretical results are in good agreement which confirms the validity of the proposed model.
\end{abstract}

\section{Introduction.}

La commande de processus industriel, la gestion de l'énergie, la surveillance des bâtiments d'habitation ou industriels nécessitent la centralisation de mesures physiques ou de données, en provenance de capteurs, vers des unités de traitement de l'information. Ces capteurs nécessitent généralement l'implantation de câbles électriques pour leur alimentation en énergie et pour la transmission des informations qu'ils fournissent [1]. Les problèmes de coût et de flexibilité nous ont conduits à utiliser un support de transmission déjà existant dans les bâtiments, les canalisations métalliques $[2,3]$, et un principe adapté à tout type de site, la transmission par couplage magnétique. A l'initiative du Centre de Recherche ELF Solaize, les premières applications concernent la gestion de l'eau chaude sanitaire en habitat collectif, la gestion du chauffage et la surveillance en habitat individuel.
La modélisation électrique que nous proposons permet d'étudier par simulation, la faisabilité et les performances de la transmission d'énergie électrique par couplage magnétique pour l'alimentation de capteurs répartis sur un réseau de canalisations.

\section{Principe de la transmission par couplage magnéti-} que.

Un réseau de canalisations d'eau chaude sanitaire ou de chauffage central étant métallique et nécessairement fermé, l'idée consiste à induire un courant dans la canalisation en appliquant les lois de l'électromagnétisme. "L'injection » d'un courant électrique dans la canalisation se fait par couplage entre un bobinage d'émission et la «boucle » constituée par la canalisation. Un bobinage de réception couplé magnétiquement et placé en un point quelconque de la boucle prélève une partie de l'énergie circulant dans la canalisation. La mise en ouvre de circuits 
magnétiques à grande perméabilité permet d'accroître le rendement de la transmission en rendant le couplage plus serré entre les circuits électriques.

\section{Choix du modèle [4].}

3.1 CAS PARTICULIER: LES BOUCleS SIMPLES DE CANALISATION. - L'étude théorique peut être menée sur une boucle simple de canalisation constituée par un tube de cuivre de diamètre, d'épaisseur et de longueur donnés. L'émetteur est constitué d'un générateur sinusoïdal $\mathrm{G}$ et le récepteur d'une charge d'impédance $\underline{Z}$. Ils sont tous les deux couplés à la canalisation par les transformateurs $T$ et $T^{\prime}$ (Fig. 1a). La boucle de canalisation constitue «l'enroulement » secondaire du transformateur $\mathrm{T}$ et l'«enroulement» primaire du transformateur $T$ '. Elle est le siège d'une force électromotrice induite qui provoque la circulation d'un courant et l'apparition de chutes de tension réparties.

Pour la modélisation, cette boucle est remplacée par un circuit électrique fermé comportant une impédance localisée $\underline{Z}_{\mathrm{C}}$. Nous avons choisi de représenter par des différences de potentiel localisées $\left(\underline{V}_{2}, \underline{V}_{\mathrm{C}}, \underline{V}_{1}^{\prime}\right)$, les tensions apparaissant le long de la boucle (Fig. 1b).

3.1.1 Cas du transformateur parfait. - La modélisation du fonctionnement de la boucle repose dans ce cas sur les hypothèses suivantes:

- les résistances des enroulements et les fuites magnétiques sont négligeables ;
- le noyau magnétique n'est pas saturé ;

- les pertes fer sont négligeables.

Les transformateurs sont donc supposés parfaits. Nous utilisons les notations suivantes :

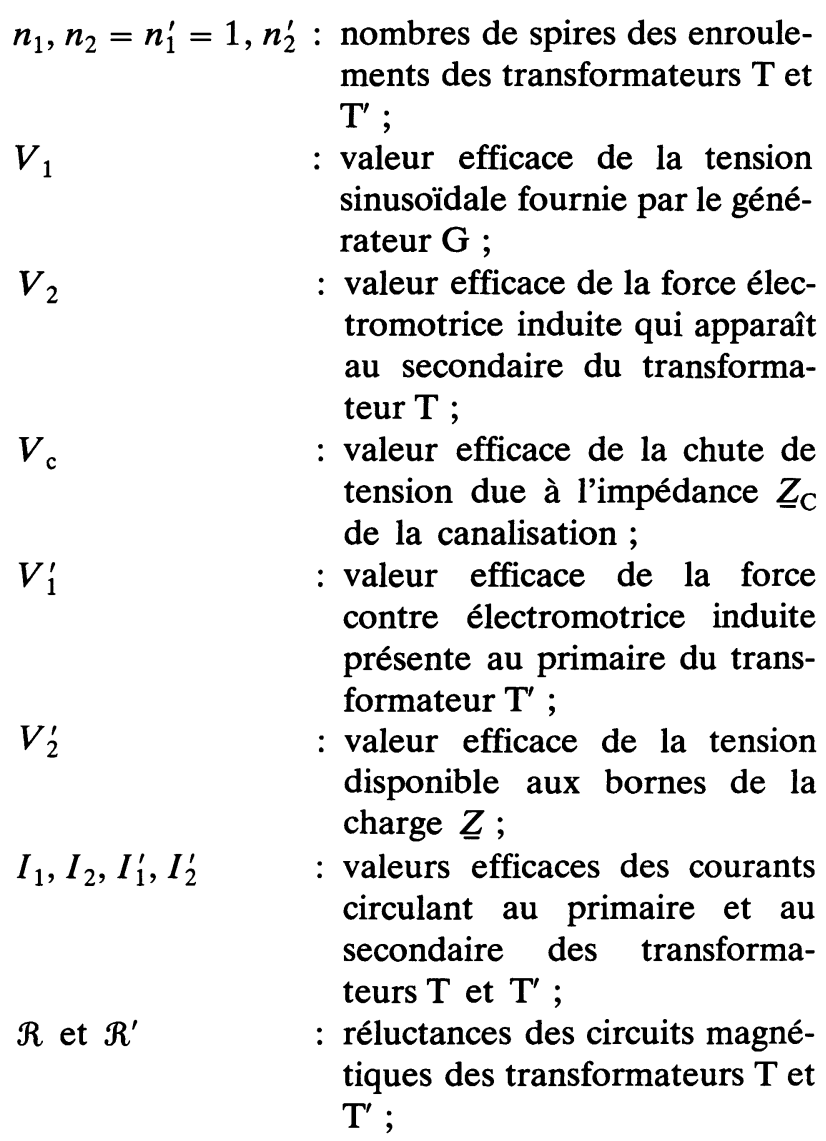

a)

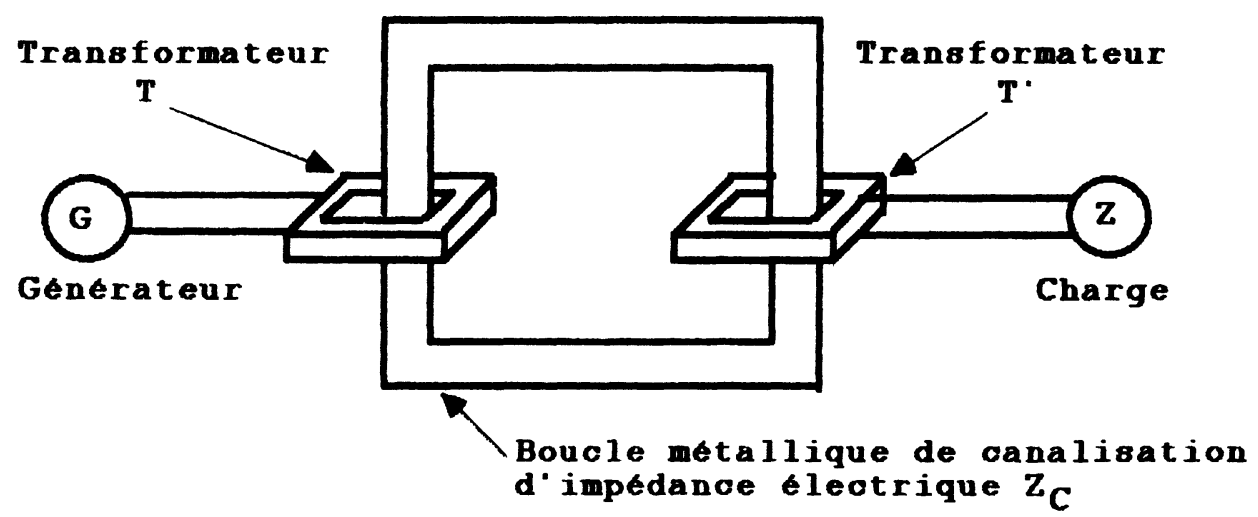

b)

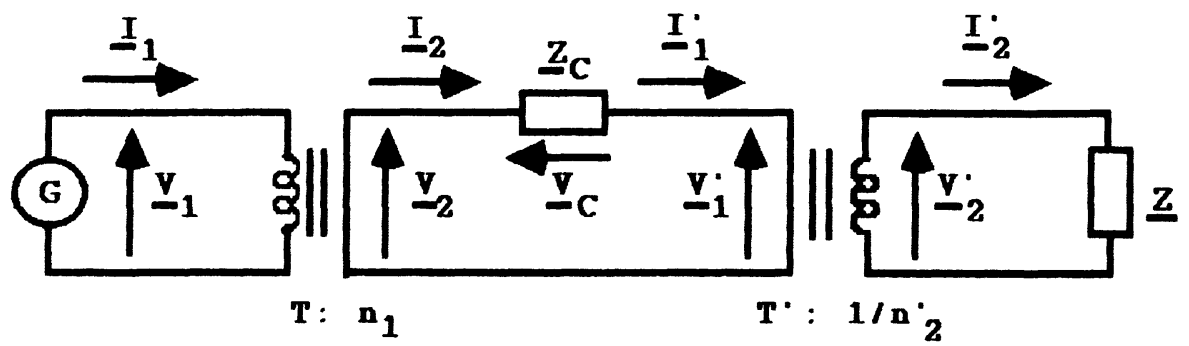

Fig. 1. - Transmission par couplage électromagnétique : (a) Principe, (b) Schéma électrique.

[Electromagnetic coupling transmission: (a) Principle, (b) Electrical circuit.] 
$\phi$

: flux circulant dans le circuit magnétique du transformateur $\mathrm{T}$.

Soient $Q_{\mathrm{T}}, Q_{\mathrm{C}}$ et ${\underline{\mathrm{T}^{\prime}}}$, les quadripôles correspondant respectivement au transformateur $\mathrm{T}$, à la boucle de canalisation et au transformateur $T^{\prime}$. Les équations classiques reliant les différentes grandeurs électriques, et les matrices de transfert associées aux quadripoles $\underline{Q}_{\mathrm{T}}, \underline{Q}_{\mathrm{C}}$ et ${\underline{\mathrm{T}^{\prime}}}_{\mathrm{T}^{\prime}}$ sont, en valeur complexe :

- Pour le transformateur $\mathrm{T}$ :

$$
\begin{aligned}
\frac{\underline{V}_{1}}{\underline{V}_{2}} & =\frac{-n_{1}}{n_{2}}=-n_{1} \\
n_{1} \underline{I}_{1}+n_{2} \underline{I}_{2} & =n_{1} \underline{I}_{1}+\underline{I}_{2}=\mathcal{R} \cdot \Phi \\
\underline{V}_{1} & =j n_{1} \omega \underline{\phi} .
\end{aligned}
$$

Des relations (1), (2) et (3), on déduit la matrice de transfert du quadripôle $\underline{Q}_{\mathrm{T}}$ équivalent au transformateur $\mathrm{T}$.

$$
\begin{aligned}
& {\left[\begin{array}{l}
\underline{V}_{1} \\
\underline{I}_{1}
\end{array}\right]=\left[\begin{array}{lr}
-n_{1} & 0 \\
-\frac{\Re}{j n_{1} \omega} & \frac{-1}{n_{1}}
\end{array}\right]\left[\begin{array}{l}
\underline{V}_{2} \\
\underline{I}_{2}
\end{array}\right]} \\
& {\left[\begin{array}{l}
\underline{V}_{1} \\
\underline{I}_{1}
\end{array}\right]=\left[\underline{Q}_{\mathrm{T}}\right] \cdot\left[\begin{array}{l}
\underline{V}_{2} \\
\underline{I}_{2}
\end{array}\right] .}
\end{aligned}
$$

- Pour la boucle de canalisation :

$$
\begin{aligned}
\underline{V}_{2} & =\underline{V}_{\mathrm{C}}+\underline{V}_{1}^{\prime}=\underline{Z}_{\mathrm{C}} \underline{I}_{1}^{\prime}+\underline{V}_{1}^{\prime} \\
\underline{I}_{2} & =\underline{I}_{1}^{\prime} .
\end{aligned}
$$

Des relations (5) et (6), on déduit la matrice de transfert du quadripôle $\underline{Q}_{\mathrm{C}}$ équivalent à la boucle de canalisation

$$
\begin{aligned}
& {\left[\begin{array}{l}
\underline{V}_{2} \\
\underline{I}_{2}
\end{array}\right]=\left[\begin{array}{ll}
1 & \underline{Z}_{\mathrm{C}} \\
0 & 1
\end{array}\right]\left[\begin{array}{l}
\underline{V}_{1}^{\prime} \\
\underline{I}_{1}^{\prime}
\end{array}\right]} \\
& {\left[\begin{array}{l}
\underline{V}_{2} \\
\underline{I}_{2}
\end{array}\right]=\left[\underline{Q}_{\mathrm{C}}\right] \cdot\left[\begin{array}{l}
\underline{V}_{1}^{\prime} \\
\underline{I}_{1}^{\prime}
\end{array}\right] .}
\end{aligned}
$$

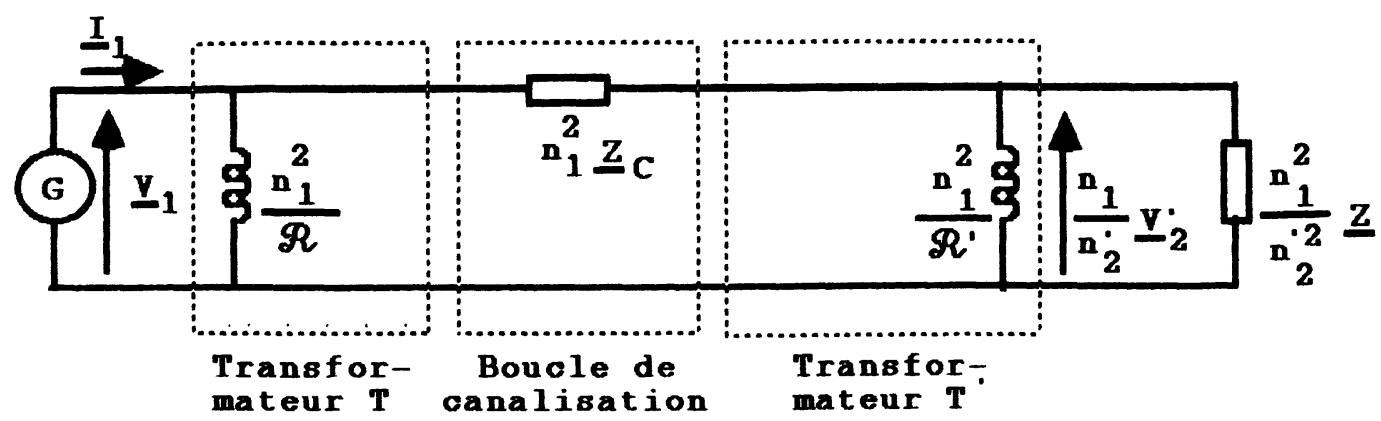

Fig. 2. - Schéma électrique équivalent.

[Equivalent electrical circuit.]
- Pour le transformateur $\mathrm{T}^{\prime}$ :

Les relations entre les grandeurs électriques sont identiques à celles obtenues pour le transformateur T. La matrice de transfert du quadripôle $Q_{\mathrm{T}^{\prime}}$ est :

$$
\begin{aligned}
& {\left[\begin{array}{l}
\underline{V}_{1}^{\prime} \\
\underline{I}_{1}^{\prime}
\end{array}\right]=\left[\begin{array}{lc}
-\frac{1}{n_{2}^{\prime}} & 0 \\
-\frac{\mathcal{R}^{\prime}}{j n_{2}^{\prime} \omega} & -n_{2}^{\prime}
\end{array}\right]\left[\begin{array}{l}
\underline{V}_{2}^{\prime} \\
\underline{I}_{2}^{\prime}
\end{array}\right]} \\
& {\left[\begin{array}{l}
\underline{V}_{1}^{\prime} \\
\underline{I}_{1}^{\prime}
\end{array}\right]=\left[\underline{Q}_{\mathrm{T}^{\prime}}\right] \cdot\left[\begin{array}{l}
\underline{V}_{2}^{\prime} \\
\underline{I}_{2}^{\prime}
\end{array}\right] .}
\end{aligned}
$$

Une installation de transmission apparaît donc comme la mise en cascade des quadripôles $Q_{\mathrm{T}}$, $Q_{\mathrm{C}}$ et ${\underline{\mathrm{T}^{\prime}}}_{\text {(Fig. 2) qui conduit à la relation matricielle }}$ suivante :

$$
\left[\begin{array}{l}
\underline{V}_{1} \\
\underline{I}_{1}
\end{array}\right]=\left[\underline{Q}_{\mathrm{T}}\right]\left[\underline{Q}_{\mathrm{C}}\right]\left[\underline{Q}_{\mathrm{T}^{\prime}}\right]\left[\begin{array}{l}
\underline{V}_{2}^{\prime} \\
\underline{I}_{2}^{\prime}
\end{array}\right] .
$$

Cette dernière relation permet, d'après l'expression de la tension aux bornes de la charge :

$$
\underline{V}_{2}^{\prime}=\underline{Z} \cdot \underline{I}_{2}^{\prime}
$$

d'obtenir la relation entre $\underline{V}_{1}$ et $\underline{I}_{1}$ :

$$
\underline{I}_{1}=\left[\frac{1}{j n_{1}^{2} \cdot \frac{\omega}{\mathcal{R}}}+\frac{1}{n_{1}^{2} \cdot \underline{Z}_{\mathrm{C}}+\frac{1}{\frac{\mathcal{R}^{\prime}}{j n_{1}^{2} \cdot \omega}+\frac{n_{2}^{\prime 2}}{n_{1}^{2} \cdot \underline{Z}}}}\right] \cdot \underline{V}_{1}
$$

et d'en déduire, l'impédance équivalente à l'ensemble du dispositif ramenée au primaire du transformateur $\mathrm{T}$.

\subsubsection{Cas réel.}

3.1.2.1 Fréquence de fonctionnement. - Afin de ne perturber ni le spectre des fréquences audibles 
$(f<20 \mathrm{kHz})$ ni le spectre des fréquences radiophoniques $(f>150 \mathrm{kHz})$, la fréquence des signaux transmis sur les réseaux de canalisations des bâtiments est choisie de l'ordre de $100 \mathrm{kHz}$.

3.1.2.2 Les circuits magnétiques. - Nous utilisons des circuits magnétiques en ferrite type U 100/57/25 en matériau $3 \mathrm{C} 8$ pour lesquels les pertes sont réduites aux fréquences élevées. D'autre part, nous choisissons de faire fonctionner les transformateurs $\mathrm{T}$ et $\mathrm{T}^{\prime}$ à induction faible de façon à ne pas saturer leurs circuits magnétiques.

3.1.2.3 Paramètres du transformateur. - Des essais réalisés sur une boucle simple de canalisation ont permis de déterminer par la méthode du diagramme de Kapp et pour chacun des deux transformateurs $T$ et $\mathrm{T}^{\prime}$, un ordre de grandeur de la résistance des enroulements et de l'inductance de fuite [5] ramenées au primaire. Ces essais nous conduisent à faire les remarques suivantes :

- A la fréquence $100 \mathrm{kHz}$, la résistance des enroulements est très faible devant l'impédance correspondant à l'inductance propre des transformateurs.

- L'inductance de fuite vaut environ le dixième de l'inductance propre des transformateurs.

Compte tenu des imprécisions sur la géométrie des réseaux de canalisations (paragraphe 3.2.3), nous avons choisi de négliger les résistances des enroulements des transformateurs $\mathrm{T}$ et $\mathrm{T}^{\prime}$, ainsi que les inductances de fuite.

De même, nous négligeons l'influence des capacités réparties des transformateurs $T$ et $T^{\prime}$ car elles n'affectent pas la transmission de puissance.
Le modèle simplifié du paragraphe 3.1.1 rend donc assez bien compte du fonctionnement réel.

\subsection{CAS GÉNÉRAL.}

3.2.1 Présentation. - Les réseaux de canalisations rencontrés sur les sites réels comportent généralement des dérivations. Les boucles simples ne sont que des cas particuliers. D'une manière générale, une installation de transmission sera représentée par les schémas blocs de la figure 3. Le problème consiste, maintenant, à déterminer la structure et les paramètres du quadripôle $\underline{Q}_{\mathrm{C}}\left(\right.$ ou $\left.n_{1}^{2} \cdot \underline{Q}_{\mathrm{C}}\right)$ à partir du modèle électrique représentant le réseau de canalisations.

3.2.2 Modélisation des transformateurs de couplage. - Les schémas des quadripôles $Q_{\mathrm{T}}$ et $n_{1}^{2} \cdot{\underline{\mathrm{T}^{\prime}}}$ sont les mêmes que dans le cas précédent puisque les systèmes de couplage du générateur et de la charge sont identiques au cas de la boucle simple.

3.2.3 Modélisation des réseaux de canalisations. L'observation de nombreuses installations de distribution d'eau chaude sanitaire et de chauffage central montre que les canalisations aller et retour d'eau chaude sont généralement disposées parallèlement et à proximité l'une de l'autre de façon à simplifier leur mise en place par l'utilisation des mêmes supports de fixation et des mêmes traversées de mur.

$\mathrm{Du}$ point de vue électrique, chaque réseau de canalisations se présente comme un ensemble de tronçons de lignes formées de deux conducteurs tubulaires parallèles (Fig. 4a) et traversés par des courants opposés. D'où l'idée de modéliser chaque tronçon compris entre deux dérivations par un

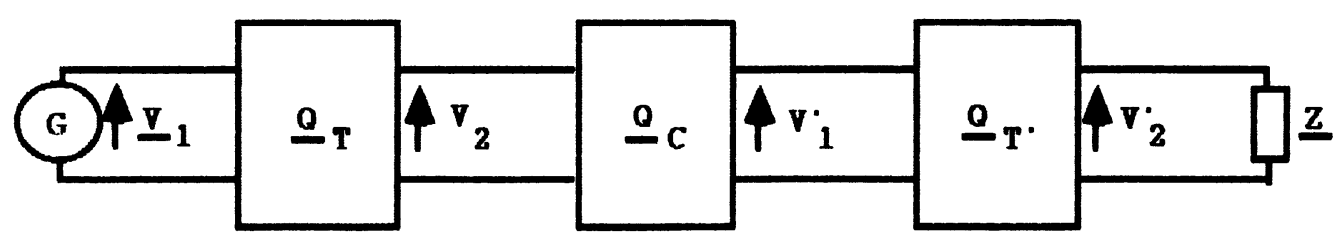

a)

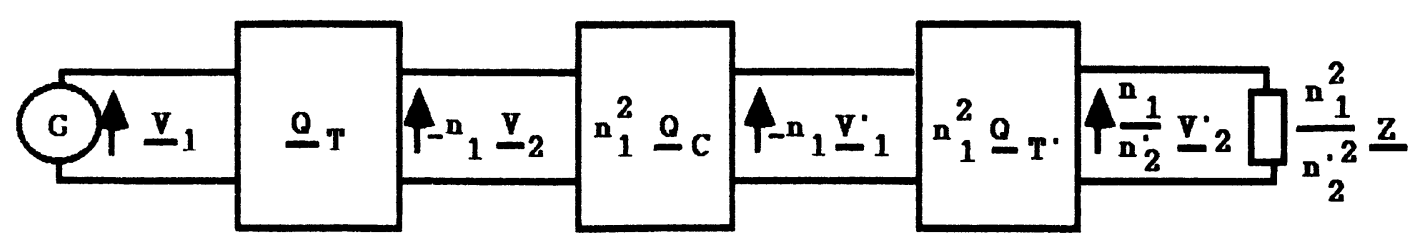

b)

Fig. 3. - Schémas blocs d'une installation de transmission: (a) Déduit de la figure 2, (b) Obtenu en ramenant les paramètres au primaire du transformateur $T$.

[Block diagrams of a transmission installation: (a) Deduced from figure 2, (b) Obtained by bringing back the parameters into the $\mathrm{T}$ transformer primary.] 
quadripôle à constantes localisées (Fig. 4b) où $R, L$, $C, G$ représentent respectivement la résistance, l'inductance, la capacité et la conductance linéiques $[6,7]$ du tronçon à la fréquence considérée.

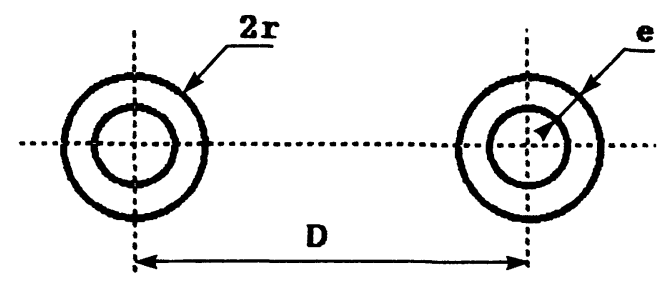

a)

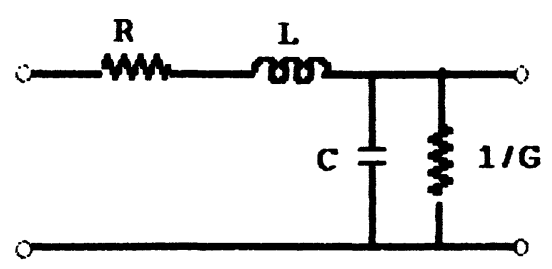

b)

Fig. 4. - Ligne électrique constituée de deux canalisations métalliques parallèles : (a) Caractéristiques géométriques, (b) Quadripôle équivalent.

[Electric line made of two parallel metallic pipes: (a) Geometrical characteristics, (b) Equivalent quadripole.]

Les paramètres électriques : résistance, inductance, capacité et conductance linéiques, à la fréquence $f=10^{5} \mathrm{~Hz}$, du quadripôle équivalent à un segment de deux conducteurs tubulaires parallèles, se calculent à partir des relations suivantes :

- Résistance des deux conducteurs lorsque $r \gg a$ et $e>a$ :

$$
R=\frac{1}{\sigma \cdot \pi \cdot r \cdot a}
$$

- Inductance interne des deux conducteurs lorsque $r \gg a$ et $e>a$ :

$$
L_{\mathrm{i}}=\frac{\mu \cdot a}{2 \cdot \pi \cdot r}
$$

- Inductance externe : $L_{\mathrm{e}}=\frac{\mu_{0}}{\pi} \cdot \ln \frac{D-r}{r}$

- Inductance totale : $L=L_{\mathrm{i}}+L_{\mathrm{e}}$

- Capacité :

$C=\frac{\pi \varepsilon_{0}}{\ln \frac{D-r}{r}}$

- Conductance :

$G=C \cdot \omega \cdot \operatorname{tg} \delta$.

avec:

$D$ : distance entre conducteurs

$r \quad$ : rayon du conducteur

$e \quad$ : épaisseur du conducteur

$\sigma \quad$ : conductivité du conducteur

$\mu \quad$ : perméabilité du conducteur

$\mu_{0}$ : perméabilité du vide

$\varepsilon_{0} \quad$ : permittivité de l'air

$f \quad$ : fréquence du courant électrique

$\operatorname{tg} \delta$ : angle de pertes du diélectrique

$a: \frac{1}{\sqrt{\pi \mu \sigma f}}:$ épaisseur de peau.

La conductance est négligeable lorsque le diélectrique est de l'air et la fréquence est inférieure au gigahertz.

Le tableau I rassemble les valeurs calculées de l'épaisseur de peau $a$ et des paramètres $R, L$ et $C$. Ces valeurs sont très voisines des valeurs mesurées ( $L=1 \mu \mathrm{H} / \mathrm{m}, C=12 \mathrm{pF} / \mathrm{m})$ sur une maquette au moyen d'un analyseur de réseau HP 4275A.

En remarquant que les dimensions pratiques des réseaux de canalisations sont toujours très inférieures à la longueur d'onde des signaux utilisés $(\lambda \# 3 \mathrm{~km})$, nous nous situons dans le cas des états quasi stationnaires. Compte tenu des ordres de grandeur des impédances $\mathrm{R}$, L $\omega$ et $1 / \mathrm{C} \omega$ (tableau II), chaque tronçon de canalisation peut être représenté, en première approximation, par une inductance

Tableau I. - Epaisseur de peau, résistance, inductance et capacité linéiques d'une ligne électrique constituée de deux canalisations métalliques parallèles : longueur $1 \mathrm{~m}$, fréquence $10^{5} \mathrm{~Hz}$, rayon $r=10^{-2} \mathrm{~m}$, épaisseur $e=10^{-3} \mathrm{~m}$, distance $D=10^{-1} \mathrm{~m}$.

[Skin effect resistance, inductance and capacitance of an electric line made of two parallel metallic pipes : length $1 \mathrm{~m}$, frequency $10^{5} \mathrm{~Hz}$, radius $r=10^{-2} \mathrm{~m}$, thickness $e=10^{-3} \mathrm{~m}$, distance $D=10^{-1} \mathrm{~m}$.]

\begin{tabular}{|l|c|c|c|c|c|c|}
\hline Matériau & $a(\mathrm{~mm})$ & $R(\Omega / \mathrm{m})$ & $L_{\mathrm{i}}(\mu \mathrm{H} / \mathrm{m})$ & $L_{\mathrm{e}}(\mu \mathrm{H} / \mathrm{m})$ & $L(\mu \mathrm{H} / \mathrm{m})$ & $C(\mathrm{pF} / \mathrm{m})$ \\
\cline { 1 - 5 } Cuivre & 0,21 & $2,6 \times 10^{-3}$ & $4,2 \times 10^{-3}$ & & & \multirow{2}{*}{0} \\
\cline { 1 - 5 } Laiton & 0,4 & $5 \times 10^{-3}$ & $8 \times 10^{-3}$ & 0,9 & 0,9 & 12,6 \\
\cline { 1 - 5 } Fer & 0,047 & $0,48 \times 10^{-3}$ & $0,94 \times 10^{-3}$ & & & \\
\hline
\end{tabular}


Tableau II. - Impédances correspondant aux paramètres $R, L$ et $C$ d'une ligne électrique constituée de deux canalisations métalliques parallèles: longueur $1 \mathrm{~m}$, fréquence $100 \mathrm{kHz}$, rayon $r=10^{-2} \mathrm{~m}$, épaisseur $e=10^{-3} \mathrm{~m}$, distance $D=10^{1} \mathrm{~m}$.

[Impedances corresponding to the $R, L$ and $C$ parameters of an electric line made of two parallel metallic pipes: length $1 \mathrm{~m}$, frequency $100 \mathrm{kHz}$, radius $r=10^{-2} \mathrm{~m}$, thickness $e=10^{-3} \mathrm{~m}$, distance $\left.D=10^{-1} \mathrm{~m}.\right]$

\begin{tabular}{|l|r|r|r|}
\hline Matériau & $R(\Omega / \mathrm{m})$ & $L \omega(\Omega / \mathrm{m})$ & $\frac{1}{C \omega}(\Omega / \mathrm{m})$ \\
\cline { 1 - 2 } Cuivre & $2,6 \times 10^{-3}$ & & \\
\cline { 1 - 2 } Laiton & $5 \times 10^{-3}$ & 0,57 & $1,26 \times 10^{5}$ \\
\cline { 1 - 2 } Fer & $0,48 \times 10^{-3}$ & & \\
\hline
\end{tabular}

proportionnelle à sa longueur. La variation logarithmique de l'inductance linéique en fonction des dimensions géométriques rend le choix de $\mathrm{r}$ et $\mathrm{D}$ peu critique. Une valeur d'inductance $L=1 \mu \mathrm{H} / \mathrm{m}$ donne généralement de bons résultats.

Un réseau de canalisations est donc représenté par un réseau électrique maillé où chaque dérivation est assimilable à une inductance. Le quadripôle $Q_{\mathrm{C}}$ de la figure 3 qui modélise l'ensemble du réseau de canalisations sera alors uniquement constitué d'inductances. Les courants dans le réseau se partageront de façon inversement proportionnelle à la réactance présentée par chaque dérivation.

Un développement analytique complet de chaque installation est envisageable mais nous avons préféré, du fait de sa souplesse, une étude menée au moyen d'un modèle paramétrable et d'une simulation sur microordinateur.

\section{Applications et résultats.}

4.1 GÉnÉralités. - Compte tenu des applications développées, nous présentons ci-dessous les résultats concernant le réseau d'eau sanitaire d'un immeuble et le réseau de chauffage d'une maison individuelle. Dans chacun des deux cas, nous avons effectué la détermination des paramètres du modèle, la simulation sur un microordinateur de la répartition des courants induits et le calcul de la puissance transmise en différents points du réseau. La simulation est réalisée au moyen du logiciel MICROCAP [8] qui permet notamment le dessin de circuits électriques analogiques et l'analyse de leur réponse temporelle et fréquentielle.

Pour mettre en œuvre le réseau de capteurs intelligents [9], dénommé TUBENET, décrit suc- cinctement au paragraphe 4.4 et faciliter la réalisation des circuits électroniques d'émission et donc de minimiser leur coût, nous avons choisi de générer un signal de tension de forme rectangulaire. Pour la simulation du fonctionnement de l'ensemble nous avons utilisé le même signal de tension.

\subsection{RÉSEAU D'EAU SANITAIRE D'UN IMMEUBLE.}

4.2.1 Détermination des paramètres. - La partie de réseau concernée est constituée d'une colonne de distribution d'eau chaude et d'une colonne de distribution d'eau froide comportant à chaque étage deux dérivations (une par appartement), représentées par des traits pointillés sur la figure 5a. De plus, elle est bouclée du fait de la présence de barres métalliques de fixation. A l'aide des résultats du paragraphe 3, on obtient le modèle électrique de la figure $5 \mathrm{~b}$.

4.2.2 Répartition des courants induits. - Le générateur de signaux carrés, d'amplitude crête à crête $V_{\mathrm{g}}=56 \mathrm{~V}$ et de fréquence $100 \mathrm{kHz}$, est couplé au point $\mathrm{A}$ du réseau de canalisations (sous-sol) par l'intermédiaire du transformateur $\mathrm{T}$ comprenant $n_{1}=10$ spires et d'inductance $L_{\mathrm{T}}=500 \mu \mathrm{H}$. La charge de cette source est le circuit équivalent représenté figure 6 . Au niveau de la simulation, des résistances $r=1 \Omega$ sont insérées dans chaque branche pour la détermination des courants (Fig. 7). La valeur de ces résistances est faible devant les valeurs de réactances (ex. : $n_{1}^{3}, L_{2} \omega \# 377 \Omega$ ). Les valeurs numériques des différents éléments sont les paramètres fournis à la simulation.

Afin de valider les résultats de la simulation, nous avons relevé directement sur site réel, à l'aide d'un capteur de mesure, les valeurs crête à crête $I$ des courants induits en plusieurs points du réseau. Pour une inductance, l'expression liant le courant $i$ la traversant à la tension $u$ à ses bornes :

$$
i=\frac{1}{L} \int u \mathrm{~d} t
$$

implique que le courant ait une forme triangulaire puisque $u(t)$ est un signal rectangulaire périodique (Fig. 8). Les harmoniques d'un signal triangulaire s'atténuant rapidement avec la fréquence (loi en $\left.1 / f^{2}\right)$, l'hypothèse de états quasi stationnaires reste valable et les perturbations apportées dans la gamme des fréquences radiophoniques sont faibles.

Le tableau III présente une comparaison entre les résultats obtenus par la simulation et ceux relevés expérimentalement. Il montre que l'intensité mesurée décroît rapidement avec les étages.

4.2.3 Puissance télétransmise. - Couplons en un point quelconque du réseau par un transformateur $\mathrm{T}^{\prime}\left(n_{2}^{\prime}=10\right.$ ou 100 spires), une charge constituée d'un dispositif redresseur et d'un condensateur $C=1000 \mu \mathrm{F}$ (Fig. 9). Ce circuit placé dans chaque 


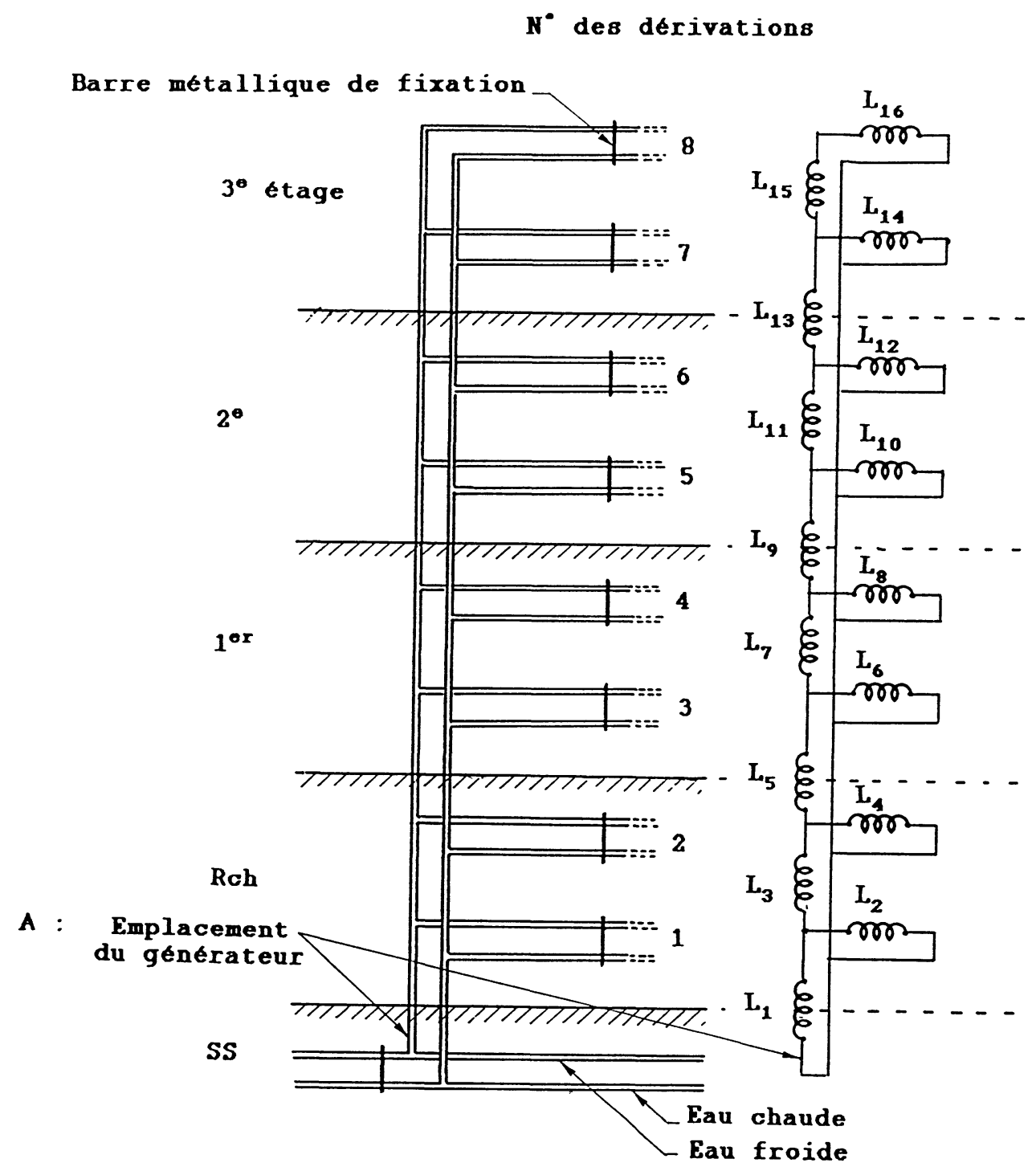

Fig. 5. - (a) Réseau d'eau sanitaire, (b) Modélisation électrique : $L_{1}=L_{3}=L_{5}=\cdots=L_{15}=1 \mu \mathrm{H}, \quad L_{2}=$ $L_{4}=L_{6}=\cdots=L_{16}=6 \mu \mathrm{H}$.

[(a) Sanitary water network, (b) Electrical modelling: $L_{1}=L_{3}=L_{5}=\cdots=L_{15}=1 \mu \mathrm{H}$, $L_{2}=L_{4}=L_{6}=\cdots=L_{16}=6 \mu \mathrm{H}$.]

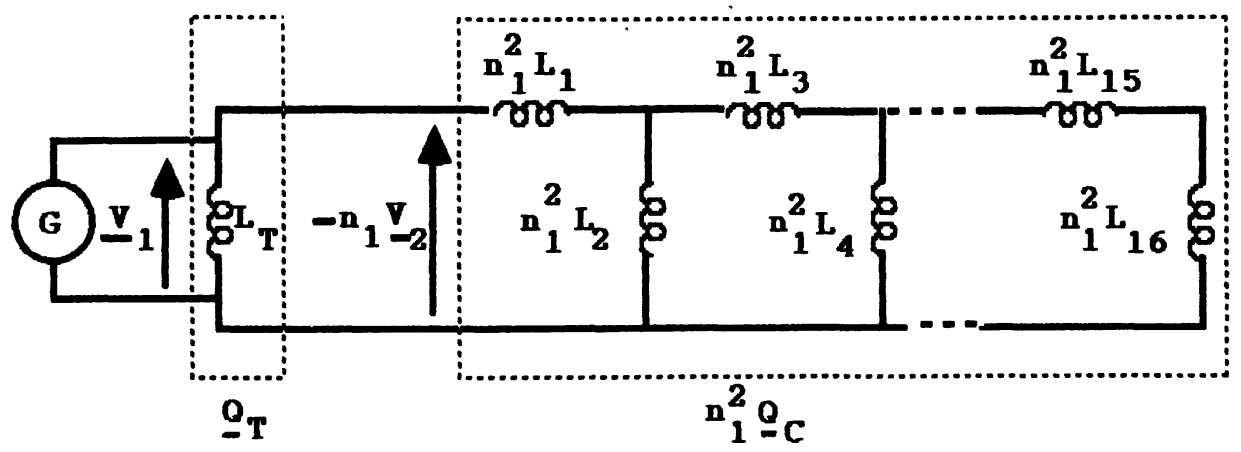

Fig. 6. - Schéma équivalent du réseau d'eau sanitaire.

[Equivalent circuit of the sanitary water network.] 


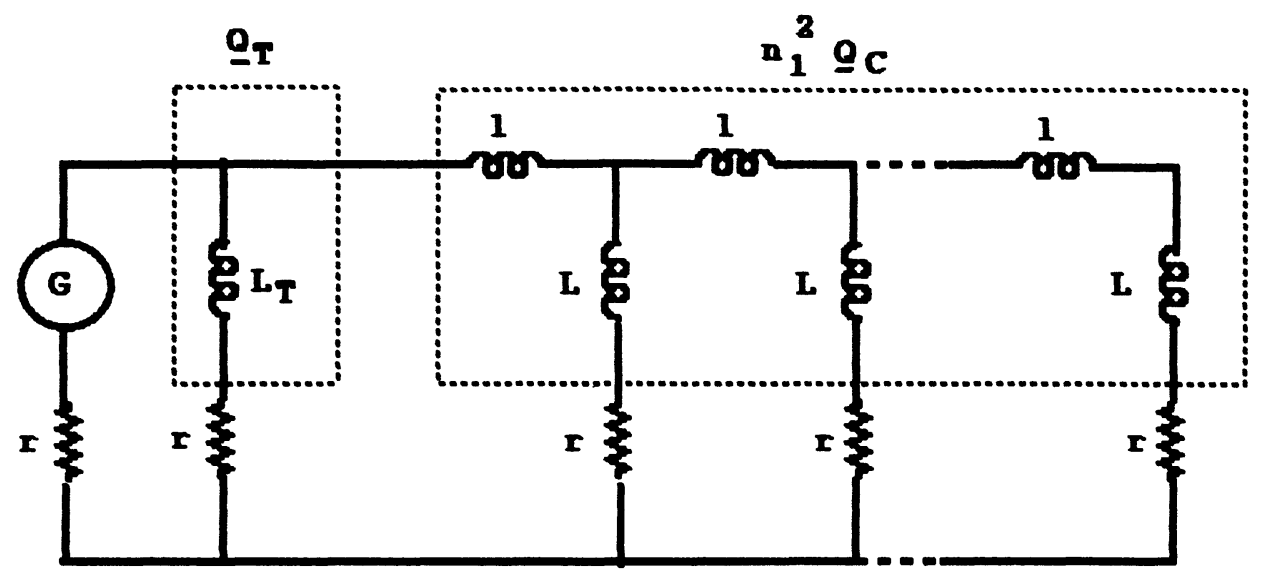

Fig. 7. - Paramètres de simulation : $r=1 \Omega, L_{\mathrm{T}}=0,5 \mathrm{mH}, L=n_{1}^{2} \cdot L_{2 \mathrm{p}}=0,6 \mathrm{mH}, \ell=n_{1}^{2} \cdot L_{2 \mathrm{p}+1}=0,1 \mathrm{mH}$. [Simulation parameters : $r=1 \Omega, L_{\mathrm{T}}=0,5 \mathrm{mH}, L=n_{1}^{2} \cdot L_{2 \mathrm{p}}=0,6 \mathrm{mH}, \ell=n_{1}^{2} \cdot L_{2 \mathrm{p}+1}=0,1 \mathrm{mH}$.]

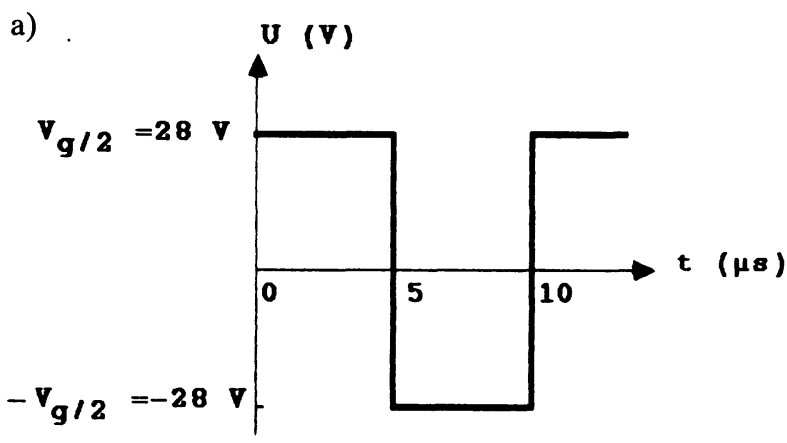

b)

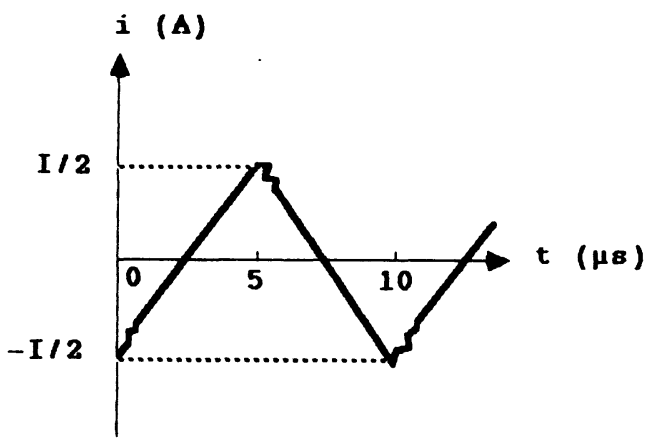

Fig. 8. - (a) Tension $u$ délivrée par le générateur, (b) Courant $i$ dans chaque branche du réseau.

[(a) Primary voltage $u$ delivered by the generator, (b) Current $i$ in each pipe of the network.]

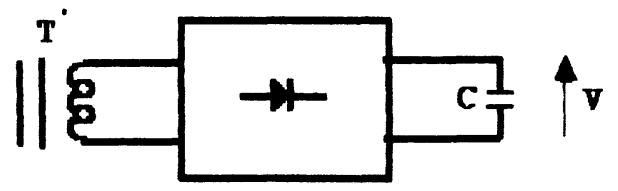

Fig. 9. - Schéma de principe du circuit de stockage de l'énergie assurant l'alimentation autonome du capteur.

[Principle diagram of the power storage circuit providing the self supply of sensors.] capteur du système TUBENET permet le stockage de l'énergie télétransmise par le poste central et assure l'alimentation autonome du capteur.

La tension induite apparaissant aux bornes du secondaire du transformateur $\mathrm{T}^{\prime}$, après avoir été redressée, charge le condensateur $\mathrm{C}$ avec une constante de temps $\tau=R_{\mathrm{T}} \cdot C$, où la résistance $R_{\mathrm{T}}$ inclut les résistances directes des diodes du redresseur, les résistances des enroulements et de la canalisation, et la résistance de sortie du générateur. Au bout de la constante de temps $\tau$, la tension aux bornes du condensateur est $V_{\tau}$ et l'énergie emmagasinée est $W=1 / 2 C V_{\tau}^{2}$. On convient d'appeler puissance télétransmise $P$, le rapport de l'énergie $W$ emmagasinée dans le condensateur et de la constante de temps $\tau$ d'où :

$$
P=\frac{W}{\tau}=\frac{C \cdot V_{\tau}^{2}}{2 \cdot \tau}
$$

Les valeurs de la tension $V_{\tau}$ disponibles aux bornes du condensateur et les puissances télétransmises $P$, obtenues à partir soit d'une simulation, soit de mesures, sont réunies dans le tableau IV. Nous observons une bonne concordance entre résultats théoriques et expérimentaux. La puissance décroît rapidement avec les étages mais reste toutefois suffisante pour téléalimenter des dispositifs à faible consommation.

\subsection{RÉSEAU DE CHAUfFAGE D'UNE MAISON INDIVI- DUELLE.}

4.3.1 Détermination des paramètres. - L'installation se compose de deux canalisations parallèles de diamètre $40 \mathrm{~mm}$ (départ et retour eau chaude) sur lesquelles sont raccordés, par des canalisations de diamètre $20 \mathrm{~mm}$, douze radiateurs. Les valeurs d'inductances du circuit électrique équivalent, qui 
Tableau III. - Comparaison entre les courants simulés et mesurés pour le réseau d'eau sanitaire. [Comparison between simulated and measured currents for the sanitary water network.]

\begin{tabular}{|c|c|c|c|c|}
\hline \multirow[t]{2}{*}{ Etage } & \multirow[t]{2}{*}{$\begin{array}{c}\mathbf{N}^{\circ} \text { de la } \\
\text { dérivation }\end{array}$} & \multicolumn{2}{|c|}{$\begin{array}{l}\text { Courant } I \text { (A) } \\
\left(V_{3}=56 \mathrm{~V}\right)\end{array}$} & \multirow{2}{*}{$\begin{array}{c}\text { Affaiblissement } \\
\text { mesuré : } \\
I / I_{\mathrm{M}} \text { en dB } \\
\left(I_{\mathrm{M}}=6,8 \mathrm{~A}\right)\end{array}$} \\
\hline & & simulé & mesuré & \\
\hline $\mathrm{RdC}$ & $\begin{array}{c}\text { Point A } \\
1 \\
2\end{array}$ & $\begin{array}{l}6,3 \\
2,1 \\
1,4\end{array}$ & $\begin{array}{l}6,8 \\
2,2 \\
1,5\end{array}$ & $\begin{array}{c}0 \\
-\quad 9,8 \\
-13,1\end{array}$ \\
\hline $1^{\mathrm{er}}$ & $\begin{array}{l}3 \\
4\end{array}$ & $\begin{array}{l}0,9 \\
0,6\end{array}$ & $\begin{array}{l}1,2 \\
0,6\end{array}$ & $\begin{array}{l}-15,1 \\
-21,1\end{array}$ \\
\hline $2^{\mathrm{e}}$ & $\begin{array}{l}5 \\
6\end{array}$ & $\begin{array}{l}0,4 \\
0,3\end{array}$ & $\begin{array}{l}0,45 \\
0,4\end{array}$ & $\begin{array}{l}-23,6 \\
-24,6\end{array}$ \\
\hline $3^{e}$ & $\begin{array}{l}7 \\
8\end{array}$ & $\begin{array}{l}0,2 \\
0,1\end{array}$ & $\begin{array}{l}0,28 \\
0,17\end{array}$ & $\begin{array}{l}-27,7 \\
-32\end{array}$ \\
\hline
\end{tabular}

Tableau IV. - Réseau d'eau sanitaire: Détermination des puissances transmises à partir des résultats de simulation ou d'expériences.

[Sanitary water network : Transmitted power determination according to simulated or experimental results.]

\begin{tabular}{|c|c|c|c|c|c|c|}
\hline \multirow{2}{*}{$\begin{array}{l}\text { Nombre de } \\
\text { spires } \\
n_{2}^{\prime}\end{array}$} & \multirow[t]{2}{*}{ Etage } & \multirow[t]{2}{*}{$\begin{array}{l}\mathrm{N}^{\circ} \text { de la } \\
\text { dérivation }\end{array}$} & \multicolumn{2}{|c|}{ Tension $\dot{V}_{\tau}(\mathrm{V})$} & \multicolumn{2}{|c|}{$\begin{array}{l}\text { Puissance télétrans- } \\
\text { mise } P(\mathrm{~mW}) \\
\text { calculée à partir }\end{array}$} \\
\hline & & & simulée & mesurée & $\begin{array}{c}\text { de la } \\
\text { simulation }\end{array}$ & $\begin{array}{c}\text { des } \\
\text { mesures }\end{array}$ \\
\hline \multirow{3}{*}{10} & $\mathrm{RdC}$ & $\begin{array}{l}1 \\
2\end{array}$ & $\begin{array}{l}30 \\
20\end{array}$ & $\begin{array}{l}32 \\
22\end{array}$ & $\begin{array}{l}450 \\
200\end{array}$ & $\begin{array}{l}510 \\
240\end{array}$ \\
\hline & 1 & $\begin{array}{l}3 \\
4\end{array}$ & $\begin{array}{l}13 \\
8,5\end{array}$ & $\begin{array}{r}17,5 \\
8,5\end{array}$ & $\begin{array}{l}85 \\
36\end{array}$ & $\begin{array}{r}150 \\
36\end{array}$ \\
\hline & 2 & 5 & 6 & 6,5 & 18 & 21 \\
\hline \multirow{2}{*}{100} & 2 & 6 & 45 & 60 & 10 & 18 \\
\hline & 3 & $\begin{array}{l}7 \\
8\end{array}$ & $\begin{array}{l}30 \\
15\end{array}$ & $\begin{array}{l}40 \\
25\end{array}$ & $\begin{array}{l}4,5 \\
1,2\end{array}$ & $\begin{array}{l}8 \\
3\end{array}$ \\
\hline
\end{tabular}

sont nécessaires à la simulation, sont déterminées conformément à la méthode du paragraphe 3 (Fig. 10).

4.3.2 Répartition des courants et puissances télétransmises. - Pour cette étude, le générateur est couplé à la branche de canalisation comportant la chaudière. Cet emplacement semble le plus judicieux pour la centralisation des mesures. La charge de cette source est le circuit équivalent représenté figure 11.

Le tableau V présente, pour le courant et la puissance télétransmise, les résultats obtenus par la simulation et les relevés expérimentaux. Il montre, comme précédemment, que les résultats de la simulation sont proches des résultats expérimentaux. 


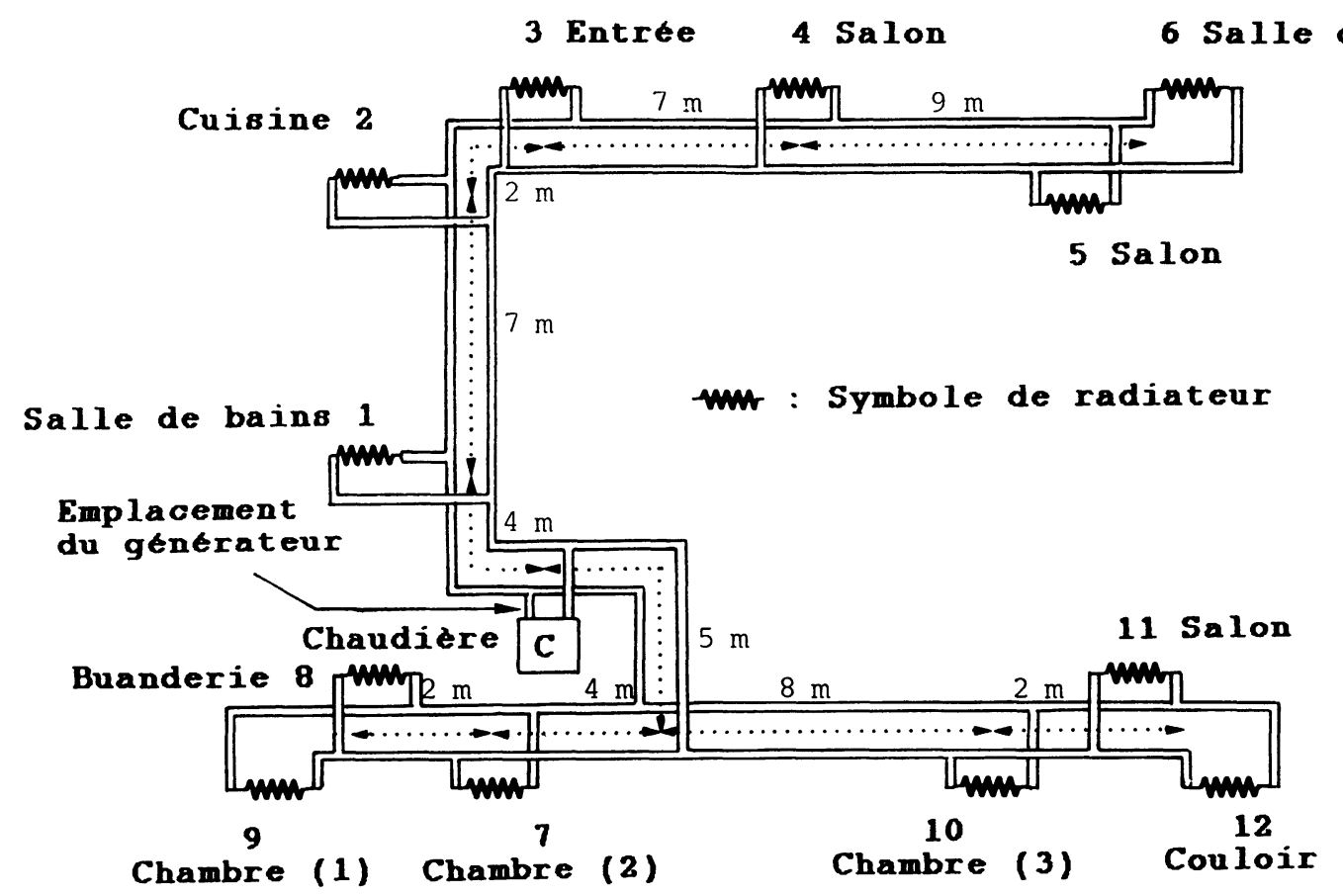

a)

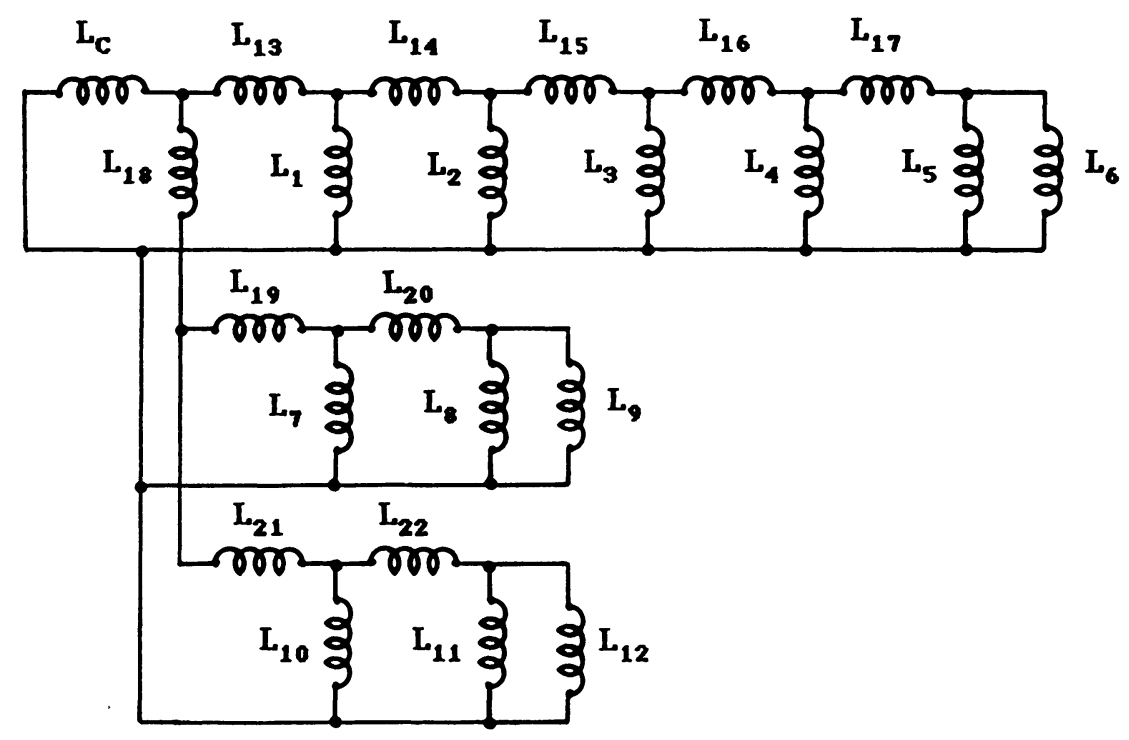

b)

Fig. 10. - (a) Réseau de chauffage central, (b) modélisation électrique :

$L_{17}=9 \mu \mathrm{H}, \quad L_{1}=L_{2}=L_{3}=\cdots=L_{12}=L_{21}=8 \mu \mathrm{H}, L_{14}=L_{16}=7 \mu \mathrm{H}$, $L_{18}=5 \mu \mathrm{H}, \quad L_{13}=L_{19}=4 \mu \mathrm{H}, \quad L_{15}=L_{20}=L_{22}=2 \mu \mathrm{H}, \quad L_{\mathrm{C}} \ll 1 \mu \mathrm{H}$.

[(a) Central heating network, (b) Electrical modelling:

$L_{17}=9 \mu \mathrm{H}, \quad L_{1}=L_{2}=L_{3}=\cdots=L_{12}=L_{21}=8 \mu \mathrm{H}, L_{14}=L_{16}=7 \mu \mathrm{H}$,

$L_{18}=5 \mu \mathrm{H}, \quad L_{13}=L_{19}=4 \mu \mathrm{H}, \quad L_{15}=L_{20}=L_{22}=2 \mu \mathrm{H}, L_{\mathrm{C}} \ll 1 \mu \mathrm{H}$.] 
Tableau V. - Répartition des courants et des puissances dans le réseau de chauffage central. [Currents and powers repartition in the central heating network.]

\begin{tabular}{|c|c|c|c|c|}
\hline \multirow{2}{*}{$\begin{array}{c}\text { Canalisation } \\
\text { comportant : }\end{array}$} & \multicolumn{2}{|c|}{$\begin{array}{c}\text { Courant } I(\mathrm{~A}) \\
\left(V_{\mathrm{g}}=84 \mathrm{~V}\right)\end{array}$} & \multicolumn{2}{c|}{$\begin{array}{c}\text { Puissance télétransmise } \\
(\mathrm{mW}) \text { calculée à partir de : }\end{array}$} \\
\cline { 2 - 5 } & simulation & mesures & simulation & mesures \\
\hline Chaudière & 7,9 & 8 & - & - \\
Radiateur 1 & 2 & 2 & 420 & 420 \\
2 & 1,1 & 1,25 & 130 & 160 \\
3 & 0,8 & 0,8 & 70 & 70 \\
4 & 0,3 & 0,25 & 10 & 7 \\
5 & 0,1 & 0,075 & 1,2 & 0,8 \\
6 & 0,1 & 0,075 & 1,2 & 0,8 \\
& & & 85 & 98 \\
8 & 0,9 & 1 & 36 & 36 \\
9 & 0,6 & 0,6 & 36 & 36 \\
10 & 0,6 & 0,6 & 36 & 18 \\
11 & 0,6 & 0,6 & 18 & 18 \\
12 & 0,4 & 0,4 & 18 & \\
\hline
\end{tabular}

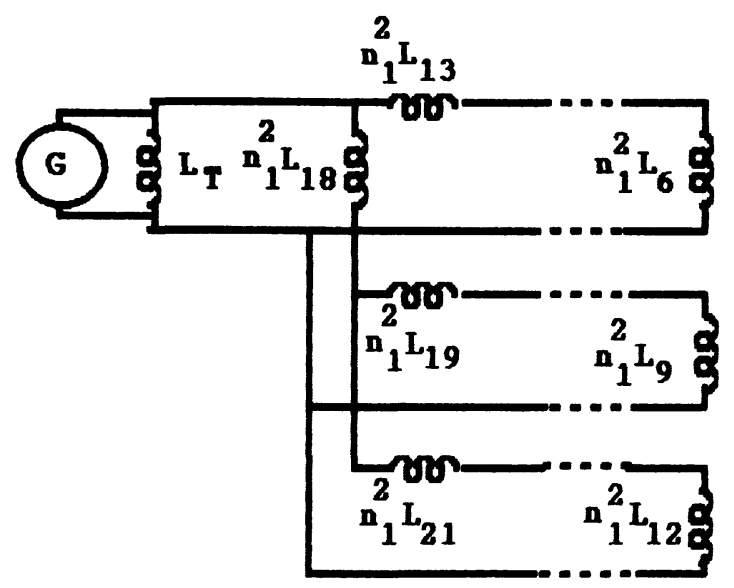

Fig. 11. - Schéma équivalent du réseau de chauffage central.

[Equivalent circuit of the central heating network.]

4.4 DÉVELOPPEMENTS INDUSTRIELS. - Les valeurs de la puissance télétransmise dans les deux applications précédentes montrent qu'il est possible d'alimenter à distance des capteurs intelligents à faible consommation [10].

Ce résultat s'est concrétisé par la réalisation du système TUBENET [11], véritable « réseau de terrain " [12] utilisant comme ligne d'alimentation en énergie mais aussi comme ligne de transmission, les réseaux de canalisations métalliques (Fig. 12). Un poste central, alimenté par le secteur $220 \mathrm{~V}$, joue le rôle de distributeur d'énergie, d'arbitre de la communication et d'unité de traitement. Les capteurs intelligents, bâtis autour de microcontrôleurs [13] à faible consommation, ne disposent que de l'énergie télétransmise par le poste central (ni pile, ni batterie). Le couplage magnétique du poste central et des capteurs confère à l'ensemble une grande flexibilité.

\section{Conclusion.}

Cette étude montre que le principe de transmission par couplage magnétique sur les réseaux de canalisations métalliques d'un bâtiment autorise la transmission à distance de l'énergie nécessaire à l'alimentation de capteurs.

Le modèle électrique proposé et la simulation développée sur micro-ordinateur permettent de prévoir la répartition des courants et des puissances télétransmises pour chaque réseau de canalisations rencontré. Les deux cas pratiques présentés montrent que la précision des résultats de simulation dépend surtout du degré de connaissance du réseau et donc des valeurs numériques des paramètres du modèle.

Le principe, le modèle et la simulation ont été utilisés pour la réalisation d'un réseau expérimental de capteurs intelligents, appelé TUBENET, où les lignes d'alimentation mais aussi de transmission sont constituées par les canalisations métalliques d'un bâtiment. 


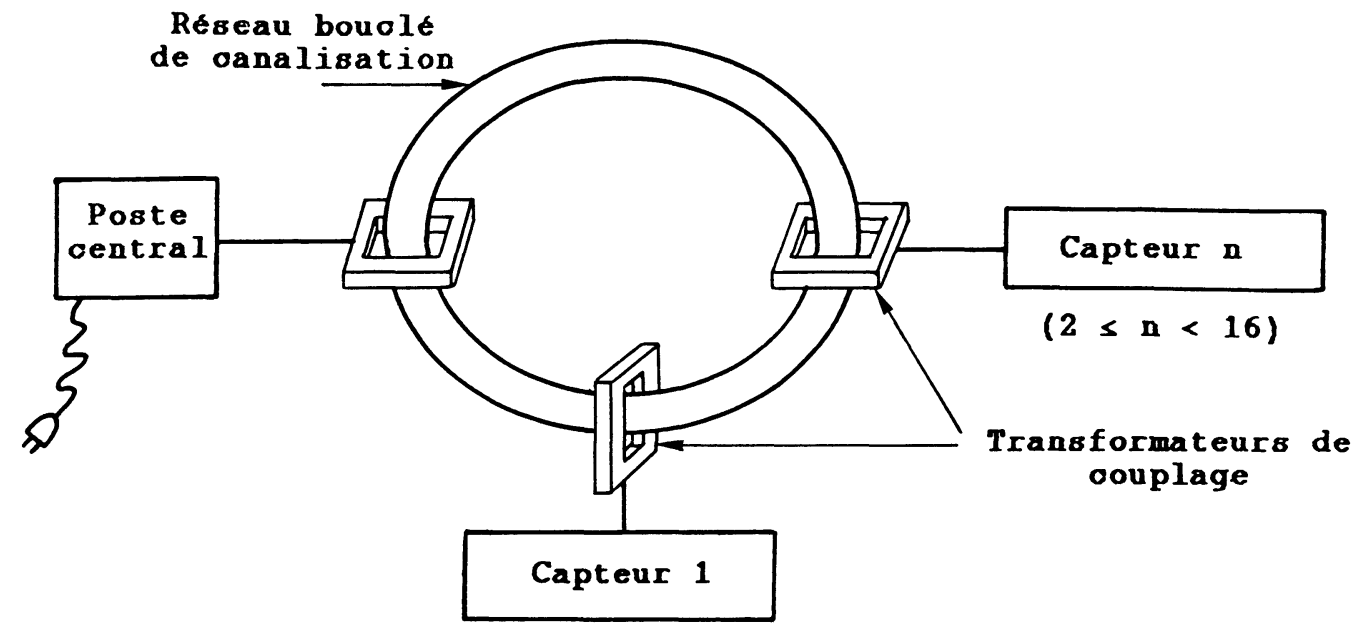

Fig. 12. - Schéma de principe du système TUBENET.

[Principle diagram of the TUBENET system.]

\section{Bibliographie}

[1] Pujolle G., Seret D., Dromard D., Horlait E., Réseaux et télématique tome II (Paris, Eyrolles) 1985.

[2] Konev K. A., Sulimin V. D., Schelkunov J. N. (Institut de recherche scientifique pour le gaz naturel de l'U.R.S.S.), Système de télécontrôle du fonctionnement des installations de ligne de pipe-lines, Brevet $n^{\circ} 2408788$, France (1977).

[3] KRAUSE G., Anordnung zur Übertragung von Signalen innerhalb von Gebäuden, Offenlegungsschrift 2829 302, Bundesrepublik Deutschland (1978).'

[4] Pellerin D., Etude et réalisation d'un système de transmission d'informations dans les bâtiments par couplage électromagnétique sur les réseaux de canalisations métalliques, Thèse de Doctorat, Lyon (1988).

[5] Guilbert A., Circuits magnétiques à flux alternatif, Transformateurs (Paris, Eyrolles) 1973.

[6] Metzger G., VABre J. P., Circuits à éléments répartis, Electronique des impulsions tome II (Paris, Masson) 1975.

[7] BonNEFILE R., Réseaux électriques linéaires à constantes réparties, Techniques de l'ingénieur (1978).
[8] Thomson A. V., Logiciel Microcomputer Circuit Analysis Program (Sunnyvale, California) 1983.

[9] FAVEnNEC J. M., Les capteurs intelligents, livre blanc CIAME-AFCET, Paris (1987).

[10] Robin J. F., Grange G., Noterman D., Brissaud M., Pellerin D., Aubert J. P., Caramelle P., Louail G., TAIRraz F., Dispositif d'alimentation à distance d'éléments de régulation tels que des actionneurs ou des capteurs émetteurs, Brevet $n^{\circ} 2574$ 196, France (1984).

[11] Pellerin D., Grange G., Robin J. F., Etude et réalisation d'un système de transmission d'informations par couplage électromagnétique sur les canalisations métalliques d'un bâtiment, $3^{\mathrm{e}}$ colloque université-industrie (C.E.E.-C.F.E.) : l'Induction Electromagnétique, Saint Nazaire (1988).

[12] Thomesse J. P., Gault M., Lerare J. P., Mesbah M., FIP : une proposition de standard de bus de terrain, Rev. Gen. Electr. 11 (1987).

[13] Al-Khalili A. J., Al-Khalili D. M., Khassem M.S., Multiple single-chip microcomputer approach to fire detection and monitoring, I.E.E. proceedings, $135 \mathrm{Pt} \mathrm{G}$ (1988) $\mathrm{N}^{\circ} 1$. 